\title{
Limitations of EBV-PCR monitoring to detect EBV associated post-transplant lymphoproliferative disorder
}

\begin{abstract}
Axelrod DA, Holmes R, Thomas SE, Magee JC. Limitations of EBV-PCR monitoring to detect EBV associated post-transplant lymphoproliferative disorder.

Pediatr Transplantation 2003: 7: 223-227. (C) 2003 Blackwell Munksgaard

Abstract: Post-transplant lymphoproliferative disorder (PTLD) represents a significant threat to the survival of pediatric transplant recipients. Epstein-Barr (EBV) viral load monitoring using polymerase chain reaction (PCR) has been reported to have a variable sensitively with relatively higher specificity as in an indicator of the development of PTLD. We report two cases of pathologically confirmed PTLD in children who failed to develop sustained increases in their EBV-PCR determined viral loads. We suggest that clinicians should be aware of the potential for false-negative results of EBV-PCR in pediatric transplant recipients.
\end{abstract}

\author{
David A. Axelrod', Ronald Holmes' ${ }^{2}$, \\ Susan E. Thomas ${ }^{2}$ and John C. Magee ${ }^{1}$ \\ ${ }^{1}$ Departments of Surgery and ${ }^{2}$ Pediatrics, University \\ of Michigan Medical Center, Ann Arbor, MI, USA \\ Key words: PTLD - EBV - PCR - transplantation \\ David A. Axelrod, MD, MBA Department of Surgery, \\ University of Michigan Health System, \\ 6312 Med Sci I, 1150 W. Medical Center \\ Drive, Ann Arbor, MI, 48109-0604, USA \\ Tel.: 734-647-4844 \\ Fax: 734-647-3301 \\ E-mail: davaxe@umich.edu \\ Accepted for publication 4 March 2003
}

The development of PTLD continues to be a major cause of morbidity and mortality among pediatric patients undergoing solid organ transplant. PTLD affects approximately $2 \%$ of solid organ recipients; however, children are disproportionately affected (1). PTLD is believed to result from primary infection or re-activation of EBV among immunosuppressed organ recipients, leading to a spectrum of diseases including malignant transformation. PTLD is associated with a wide spectrum of histopathologies, ranging from early hyperplastic lesions resembling infectious mononucleosis to true B-cell lymphomas (2). In addition, transplant recipients have developed Hodgkin's disease, T-cell and null-cell lymphomas, which are currently considered within the spectrum of PTLD, although this remains somewhat controversial (2).

Children with PTLD present with non-specific symptoms including fever, lethargy or malaise,

\footnotetext{
Abbreviations: ATGAM, anti-thymocyte globulin; CMV, cytomegalovirus; EBV, Epstein-Barr Virus; LMP, latent membrane protein; MMF, mycophenolate mofetil; PBLs, peripheral blood lymphocytes; PCR, polymerase chain reaction; PET, positron emission tomography; PTLD, posttransplant lymphoproliferative disorder; RQ, real-time quantitative.
}

diarrhea, and non-specific physical signs including hepatosplenomegaly and diffuse lymphadenopathy. Clinicians have utilized serologic markers of EBV infection to assist in the diagnosis; however, seroconversion may not occur in up to $37 \%$ of pediatric patients with PTLD (1). More recently, the use of peripheral blood PCR monitoring of EBV DNA has been shown to be a sensitive and specific method of identifying children at increased risk of developing PTLD (3). While early reports using RQ-PCR in serum, suggested that PCR was $100 \%$ sensitive and $100 \%$ specific for the detection of PTLD in children with $>1000$ copies of EBV per $100 \mu \mathrm{L}$ plasma, recent studies have suggestive that monitoring is relatively less sensitive but remains fairly specific $(4,5)$. Similarly, significantly elevated viral loads were found in 10 of 10 patients with known PTLD using a TaqMan based EBV-PCR assay (6).

EBV-PCR in PBLs has been widely applied clinically in our institution and is used as an initial diagnostic evaluation in patients with suspected EBV induced PTLD (7). However, in this report, we described two patients with pathologically proven PTLD associated with EBV who had no sustained elevation in virus detected using PCR above 100 copies per $10^{5}$ lymphocytes. 


\section{Axelrod et al.}

\section{Methods}

Clinical and laboratory records were reviewed for patients who developed PTLD following solid organ transplantation. Two children were identified who developed biopsy proven PTLD, which was EBV positive based on specimen immunohistochemistry. In each case, viral load was monitored using the quantitative competitive PCR method described by Rowe and colleagues and is reported as the number of viral DNA copies per $10^{5}$ PBLs. A value of $>500$ copies per $10^{5}$ PBLs was considered to be suggestive of PTLD (3). This project was reviewed and approved by the University of Michigan Medical Center Institutional Review Board and the parents of both children authorized publication. The initials presented have been changed to further protect the anonymity of the children.

\section{Results}

Case report 1

AA was the product of a $32 \mathrm{wk}$ twin gestation who was born at $2.2 \mathrm{~kg}$. Shortly after birth, AA presented to her primary care physicians with fussiness and irritability. Laboratory studies obtained as part of an evaluation for sepsis revealed a total protein of $2.8 \mathrm{~g} / \mathrm{dL}$ and an albumin of $1.0 \mathrm{~g} / \mathrm{dL}$. AA was subsequently diagnosed with congenital nephrotic syndrome. AA was managed with dietary supplementation and i.v. albumin until 6 months of age. At this time, AA underwent bilateral nephrectomy to prevent ongoing proteinuria. AA was managed on hemodialysis for 22 days and subsequently underwent a living related renal transplant from her mother in 1993 at the University of Michigan Medical Center. AA was CMV sero-negative and EBV sero-negative and received a CMV positive organ. AA received ATGAM induction and her initial immunosuppression included prednisone, cyclosporine, and azathioprine. AA did well after transplant aside from reflux at her ureteroneocystostomy requiring collagen injections and re-implantation. AA developed a rising creatinine 36 months post-transplant. Graft biopsy was consistent with chronic cyclosporine toxicity and her immunosuppression was changed from azathioprine to MMF.

AA's clinical course remained stable until 90 months after transplantation during this which time AA was treated with growth hormone for 30 months for short stature. AA then presented with fever to $103^{\circ} \mathrm{F}$. AA was initially treated for bronchitis at an outside hospital and discharged with persistent, intermittent fevers. AA subsequently presented to the University of Michigan with fevers to $105^{\circ}$ and an elevated creatinine. No source for her fever was identified. A renal biopsy revealed chronic cyclosporine toxicity and evidence of acute tubular necrosis.
Table 1. EBV-PCR results for AA

\begin{tabular}{lcl}
\hline $\begin{array}{l}\text { Months } \\
\text { post-transplant }\end{array}$ & $\begin{array}{l}\text { EBV genomes } / 10^{5} \\
\text { lymphocytes }\end{array}$ & $\begin{array}{l}\text { WBC } \\
\left(10^{3} \text { cells/mL) }\right.\end{array}$ \\
\hline 90.8 & 0 & 10.7 \\
91.2 (PTLD.diagnosed) & 0 & 5.8 \\
91.5 & 20 & 2.5 \\
103.7 & 100 & 6.4 \\
106.1 & 20 & 7.3 \\
\hline
\end{tabular}

Her cyclosporine dosed was reduced. Additionally, serologic studies for CMV were negative. An EBV-PCR was also obtained, and no virus was detectable in the patient's peripheral lymphocytes (Table 1). AA was re-admitted 8 days later with persistent fever. A CT scan of her chest, abdomen, and pelvis demonstrated marked mediastinal and retroperitoneal lymphadenopathy with mild tracheal compression. Open lymph node biopsy of a paratracheal node revealed classic Reed-Sternberg cells and ReedSternberg cell variants in a background rich in reactive histiocytes and small lymphocytes. Immunohistochemistry revealed that the malignant cells were EBV-LMP positive. This biopsy was determined to be consistent with classical Hodgkin's disease. Repeat serum PCR obtained at the time of this biopsy was 20 genomes per $10^{5}$ lymphocytes.

Following diagnosis, MMF and Cyclosporine were withdrawn and chemotherapy initiated. AA was treated with six cycles of COPP/ABV (cytoxan, prednisone, vincristine, procarbazine, velban, bleomycin, and adriamycin). Four months after the initiation of therapy, CT scan revealed no evidence of recurrent disease. Cyclosporine was restarted 8 months following diagnosis. At the time of her most recent follow-up, AA is now $8 \mathrm{yr}$ post-transplant and 15 months after her diagnosis of PTLD. AA is without evidence of recurrence of her Hodgkin's lymphoma and her creatinine is $1.2 \mathrm{mg} / \mathrm{dL}$.

\section{Case Report 2}

$\mathrm{BB}$ was referred for liver transplant evaluation at age seven. BB was diagnosed with biliary atresia in childhood and underwent a Kasai procedure at 2 months of age. At the time of his transplant evaluation, BB had experienced several episodes of gastrointestinal bleeding as a result of his significant esophageal varicies and splenomegaly. Prior to transplant, BB was EBV sero-negative and CMV sero-negative. BB underwent orthotopic liver transplantation 3 months after evaluation with placement of an endobiliary stent. The donor was a CMV sero-negative, EBV 
Table 2. EBV-PCR results for BB

\begin{tabular}{lcc}
\hline $\begin{array}{l}\text { Months } \\
\text { post-transplant }\end{array}$ & $\begin{array}{l}\text { EBV genomes } / 10^{5} \\
\text { lymphocytes }\end{array}$ & $\begin{array}{l}\text { WBC } \\
\left(10^{3} \text { cells } / \mathrm{mL}\right)\end{array}$ \\
\hline 0.3 & 8 & 5.5 \\
0.8 & 80 & 10.6 \\
1.0 & 0 & 7.3 \\
1.9 & 100 & 8.1 \\
2.4 & 0 & 6.3 \\
2.9 & 0 & 5.4 \\
3.3 & 40 & 5.1 \\
3.8 & 40 & 8.6 \\
4.9 & 80 & 14.1 \\
5.4 (PTLD diagnosed) & 8 & 13.9 \\
5.7 & 80 & 16.4 \\
7.1 & 0 & 8.3 \\
8.0 & 0 & 4.7 \\
9.6 & 0 & 3.8 \\
\hline
\end{tabular}

sero-positive $3 \mathrm{yr}$ old. BB was started on tacrolimus, MMF, and prednisone. His post-operative course was uneventful and BB was discharged on post-operative day number five receiving viral prophylaxis with acyclovir. BB received a single dose pulse of steroids for a presumed rejection as a result of rising liver function tests, which was not confirmed by graft biopsy.

The patient was re-admitted $6 \mathrm{wk}$ after transplantation with fever to $102.5^{\circ}$ following removal of his endobilliary stent. The blood, urine and sputum cultures were obtained, but no pathologic organisms were isolated by laboratory culture. His liver function tests were unremarkable and BB was clinically asymptomatic. BB was subsequently discharged with low-grade fevers. EBV-PCR demonstrated 8 copies per $10^{5}$ lymphocytes (Table 2). Following his discharge, the patient complained of recurrent low-grade fevers and abdominal pain. Physical examination revealed tonsillar enlargement. Given these findings, a repeat EBV-PCR was obtained and there was a transient rise in his titers from 8 to 100 copies. An abdominal-pelvic CT was obtained which demonstrated a small hypodense lesion in the left lobe of the liver and multiple small areas in the parenchyma of the right kidney showing no enhancement that were suspicious but not diagnostic for PTLD. The immunosuppression was not altered given the results of the EBV-PCR. Both the CT scan and the EBV-PCR were subsequently repeated within 1 month. These repeat studies demonstrated a reduction in viral load to 40 genomes per $10^{5}$ lymphocytes and no visible lesions on CT.

BB did well for an additional 2 months when BB developed nausea, vomiting and weight loss. BB was evaluated with EGD and colonoscopy that were normal. BB was also noted to have persistent tonsillar enlargement, and underwent tonsillectomy. Pathologic examination revealed no PTLD. Three weeks following tonsillectomy, BB represented with decreased oral intake, weight loss, and repeat CT scan demonstrated irregular thickening of several small intestinal loops in the right lower quadrant consistent with PTLD. Repeat EBV-PCR revealed only 80 genomes per $10^{5}$ lymphocytes. The patient was then taken for exploratory laparoscopy $1 \mathrm{wk}$ later, which was converted, to open laparotomy. At operation, a large right lower quadrant mass involving $12 \mathrm{~cm}$ of distal ilium was resected. BB was also found to have massive mesenteric adenopathy. Pathologic examination of this mass demonstrated a diffuse large B-cell lymphoma (monomorphic PTLD). Numerous cells were positive for EBV-LMP as well as CD20. PET scanning obtained post-operatively demonstrating 11 foci of abnormally increased FDG uptake in the mid abdomen, corresponding to lymph nodes involved with his neoplasm.

Following his diagnosis, the patient's immunosuppression was withdrawn and was treated with four doses of Rituximab. Six weeks following diagnosis and withdrawal of his immunosuppression, BB had rising liver function tests. BB was biopsied and treated for acute cellular rejection. Despite initial improvement with pulse steroids, the patient's liver function has continued to worsen. Subsequent biopsy has demonstrated chronic rejection, and $\mathrm{BB}$ is now being considered for retransplantation. Following completion of therapy with Rituximab and six cycles of cytoxan, the patient's CT scan demonstrated complete regression of his tumor. Repeat PET scan demonstrated no active disease.

\section{Discussion}

The development of PTLD represents one of the most lethal complications of solid organ transplantation. The incidence of PTLD varies by organ and has been reported in $2.6-9.0 \%$ of pediatric renal transplant recipients (8-11) with malignancy developing in $1.5 \%$. Among liver recipients, PTLD has been diagnosed in $6.8-13.1 \%$ of recipients $(12,13)$. Furthermore, the incidence of PTLD appears to be increasing in pediatric renal transplant recipients from 298 to 395 cases per $100000 \mathrm{yr}$ of patient follow-up (13).

Detection of PTLD requires a high index of suspicion, particularly among patients with known risk factors including: younger age at transplant, EBV negative recipients transplanted 
with an EBV positive organ, CMV infection, OKT3 treatment, and, possibly, tacrolimus based immunosuppression. In patients suspected of having PTLD, an aggressive diagnostic evaluation with radiographic imagining and serologic evaluation is indicated as early diagnosis may be correlated with better outcome (14). Histologic evaluation of an appropriate biopsy specimen is necessary to differentiate PTLD from acute cellular rejection, which often has a similar presentation.

Given the difficulty in identifying PTLD, viral load monitoring has been a very useful addition to the diagnostic techniques. Elevated levels of EBV DNA have been correlated with PTLD in several non-quantitative assays. The presence of low levels of EBV viral DNA is not uncommon in the immunosuppressed population, and has been documented in up to $19 \%$ of healthy solid organ transplant recipients (6). The development of a quantitative PCR technique has significantly improved the specificity for PTLD and may serve as an early marker in high-risk patients. Quantitative PCR as originally described by Rowe and colleagues utilized a competitive PCR assay in which viral load was quantified by co-amplifying varying quantities of a competitor plasmid under identical conditions (3). Using this assay, 13 of 14 patients with PTLD had $>500$ copies per $10^{5}$ lymphocytes. The resulting sensitivity was reported as $92.8 \%$ and the specificity was $100 \%$ (3).

PCR has also been effectively used to monitor high risk transplant patients and identify those with an increase risk for PTLD (15). Frequent monitoring and treatment with reduction of immunosuppression and anti-viral therapy has been reported to reduce the incidence of PTLD in pediatric liver transplant patients from 10 to $5 \%$ when compared with historical controls (16). Similarly in children undergoing small bowel transplant, the use of frequent monitoring has resulted in a reduction in the incidence of PTLD from 40 to $11 \%$ (17). EBV-PCR monitoring has also proven to be a useful strategy following the treatment of PTLD. Response to therapy is correlated with a decrease in viral load to $<200$ copies per $10^{5}$ cells (18). This reduction is reflective of an effective immune response against EBV-infected B-cells and is an early predictor of clinical outcome (15). Persistence of viral loads less than this level appears to signal that re-introduction of low dose immunosuppression is safe.

While EBV-PCR clearly has a role in the clinical management of the post-transplant patient, several limitations have been recognized. First, as shown by the patients included in this report, quantitative EBV-PCR may fail to identify patients with PTLD whose viral loads fail to meet an identified threshold value. Green and colleagues described a similar child in whom PTLD with nervous system involvement was diagnosed in a patient who had a past history of systemic PTLD. This child was noted to have a low EBV load by PCR despite active disease. Second, there may be an overlap in viral levels in patients with PTLD and EBVassociated viral syndromes (19). Finally, the significance of viral levels in the post-PTLD patient remains unclear. Patients may have a rebound in their viral load following the re-introduction of immunosuppression. However, the vast majority will not develop recurrent disease (15).

It is not clear why EBV-PCR monitoring failed to identify the development of PTLD in our patients. In patient AA, the late development (91 months post-transplant) of Hodgkin's disease, an uncommon histologic subtype may be responsible. While late onset PTLD has been associated with a higher incidence of EBV negative tumors, this was not the case in this patient as her tumor was proven to be EBV positive (2). The second patient had recently received acyclovir, which may have artificially suppressed his viral load. However, the bulk of the viral load in peripheral blood in patients with PTLD is believed to be related to immortalized cells and not lytic infection [which is suppressed by anti-viral agents (19)] Furthermore, EBV viral loads may climb, even in the face of i.v. acyclovir or gancilovir (16). Thus, it appears unlikely that the acyclovir therapy is primarily responsible for the low levels of EBV documented in this case. Hopefully, the availability of rapid, accurate viral load monitoring will facilitate further research to understand the pathologic basis of PTLD in patients who do not manifest high viral loads.

In summary, we report two cases of PTLD in patients with very low viral loads. Each patient was high risk for PTLD based on his or her initial EBV serology. While our clinical suspicion remained high, the resulting low EBV-PCR may have provided a false sense of re-assurance. In both instances, the use of CT technology was diagnostic once lesions of sufficient size had developed. While EBV-PCR remains a useful screening test in patients who are at high risk for PTLD, a significant minority of patients may have active disease despite low viral loads and alternative diagnostic modalities should be utilized to insure an early, accurate diagnosis. 


\section{References}

1. Collins MH, Montone KT, Leahey AM, et al. Post-transplant lymphoproliferative disease in children. Pediatr Transpl 2001: 5: 250-257.

2. NALESNIK MA. The diverse pathology of post-transplant lymphoproliferative disorders: the importance of a standardized approach. Transpl Infect Dis 2001: 3: 88-96.

3. Rowe DT, Qu L, ReYes J, et al. Use of quantitative competitive PCR to measure Epstein-Barr virus genome load in the peripheral blood of pediatric transplant patients with lymphoproliferative disorders. J Clin Microbiol 1997: 35: 1612-1615.

4. WAgner HJ, Wessel M, JABS W. Patients at risk for development of posttransplant lymphoproliferative disorder: plasma versus peripheral mononuclear cells as material for quantification of Epstein-Barr viral load by using real-time quantitative polymerase chain reaction. Transplantation 2001: 72: $1012-1019$.

5. Green M, Bueno J, Rowe D, et al. Predictive negative value of persistent low Epstein-Barr virus viral load after intestinal transplantation in children. Transplantation 2000: 70: 593-596.

6. Niesters HG, van Esser J, Fries D, et al. Development of real-time quantitative assay for detection of Epstein-Barr virus. J Clin Microbiol 2000: 38: 712-715.

7. Holmes, RD. Sokol RJ. Epstein- Barr virus and post transplant lymphoproliferative disease. Pediatr Transpl 2002: 6: 456-464.

8. Wingen A-M, Wiesel M, Mohring K, Scharer K, Mahis O. Malignancies in children with renal replacement therapy. Transplant Proc 1994: 26: 5-6.

9. Srivastava T, Zwick D, Rothberg PG, Warady BA. Posttransplant lymphoproliferative disorder in pediatric renal transplantation. Pediatr Nephrol 1999: 13: 748-754.

10. Hebert D, Sullivan EK. Malignancy and posttransplant lymphoproliferative disorder (PTLD) in pediatric renal transplant recipients: a report of the North American Pedatric Renal Transplant Cooperative Study (NAPRTCS) (Abstract). Pediatr Transpl 1998: 2 (Suppl.1): 57.
11. Shapiro R, Scantlebury VP, Jordan ML, et al. Pediatric renal transplantation under tacrolimus-based immunosuppression. Transplantation 1999: 67: 299-303.

12. Reyes J, Jain A, Mazariegos G, et al. Long-term results after conversion from cyclosporine to tacrolimus in pediatric liver transplantation for acute and chronic rejection. Transplantation 2000: 69: 2573-2580.

13. Cox KL, Lawernece-Miyasaki LS, Garcia-Kennedy R, et al. An increased incidence of Epstein-Barr virus infection and lymphoproliferative disorder in young children on FK506 after liver transplantation. Transplantation 1995: 59: 524-529.

14. Green M, Reyes J, Webber S, Rowe D. The role of antiviral and immunoglobulin therapy in the prevention of Epstein-Barr virus infection and post-transplantation lymphoproliferative disease following solid organ transplantation. Transpl Infect Dis 2001: 3: 97-103.

15. Rowe DT, Webber S, Schauer EM, Reyes J. Green M. Epstein-Barr virus load monitoring: its role in the prevention and management of post-transplant lymphoproliferative disease. Transpl Infect Dis 2001: 3: 79-87.

16. McDiarmid SV, Jordan S, Kim GS, et al. Prevention and preemptive therapy of postransplant lymphoproliferative disease in pediatric liver recipients. Transplantation 1998: 66: 1604-1611.

17. Green M, Bueno J, Rowe D, et al. Predictive negative value of persistent low Epstein-Barr virus viral load after intestinal transplantation in children. Transplantation 2000: 70: 593-596.

18. Green M, Cacciarelli TV, Mazariegos G, et al. Serial measurement of Epstein-Barr viral load in peripheral blood in pediatric liver transplant recipients during treatment for posttransplant lymphoproliferative disease. Transplantation 1998: 66: 1641-1644.

19. Green M, Michaels MG, Webber SA, Rowe D, Reyes J. The management of Epstein-Barr virus associated post-transplant lymphoproliferative disorders in pediatric solid-organ transplant recipients. Pediatric Transpl 1999: 3: 271-281. 